\section{Irren ist menschlich: Haftung wegen Diagnose- und/oder Befunderhebungsfehlern sowie Aufklärungsfehlern im Rahmen des Mammografie-Screenings}

\section{Einführung}

Diagnostischen Eingriffen unter Einsatz von ionisierenden Strahlen - und infolgedessen insbesondere dem Mammografie-Screening - wohnen in zweierlei Richtungen erhebliche Haftungsrisiken inne: Angesichts der Komplexität und der Unbeherrschbarkeit des menschlichen Organismus' sowie zahlreicher Überschneidungen und Abgrenzungsprobleme der Krankheitsbilder ist es für den behandelnden Radiologen äußerst schwierig, eine exakte und sichere Diagnose zu stellen. In diesem Kontext schließt sich auf Grund der unterschiedlichen Anforderungen und Rechtsfolgen die schwierige Frage nach der Abgrenzung zum Befunderhebungsfehler an. Zum anderen stellt die höchstrichterliche Rechtsprechung auf Grund des fehlenden therapeutischen Eigenwertes bei gleichzeitiger Strahlenbelastung erhöhte Anforderungen an die Aufklärung des Patienten und die damit verbundenen Risiken. Dieser Beitrag zeigt daher die einschlägigen Haftungsrisiken auf und setzt sich zudem mit den Verantwortlichkeiten der beteiligten Ärzte, insbesondere des programmverantwortlichen Arztes (PVA) anhand der ergangenen Judikatur auseinander.

\section{Haftung wegen eines Behandlungsfehlers}

Gem. § 630a Abs. 2 BGB hat die Behandlung nach den zum Zeitpunkt der Behandlung bestehenden, allgemein anerkannten fachlichen Standard zu erfolgen. Geschuldet ist mithin der sog. Facharztstandard, d. h. eine Behandlung, welche durch den jeweiligen Stand der naturwissenschaftlichen Erkenntnisse und der ärztlichen Erfahrung des entsprechenden Fachgebietes repräsentiert wird, zur Erreichung des ärztlichen Behandlungsziels erforderlich ist und sich in der Erprobung bewährt hat ${ }^{1}$. Um einen Behandlungsfehler annehmen zu können, muss

1 Vgl. BT-Drs. 17/10 488, S. 19. dem behandelnden Arzt die Unterschreitung des Facharztstandards auch subjektiv vorwerfbar sein, d. h. er muss schuldhaft gehandelt haben. Insoweit gilt ein verobjektivierter Fahrlässigkeitsmaßstab ( $\$ 276$ Abs. 2 BGB). Demzufolge sind etwaige persönliche Unzulänglichkeiten des behandelnden Arztes unbeachtlich. Vielmehr ist auf den Sorgfaltsmaßstab abzustellen, den der Verkehr von einem ordentlichen, pflichtgetreuen Durchschnittsarzt der jeweiligen ärztlichen Fachgruppe in der konkreten Situation nach dem Stand der medizinischen Wissenschaft und Praxis an Kenntnissen, Wissen, Können und Aufmerksamkeit erwarten darf. Die Haftung wegen eines Behandlungsfehlers setzt demzufolge neben der Standardunterschreitung auch das Verschulden des Arztes voraus.

\section{Voraussetzungen des Diagnose- fehlers}

Im Fachgebiet der Radiologie ist der Vorwurf des Diagnosefehlers von besonderer Bedeutung. Wie bereits eingangs erwähnt, ist es aufgrund der zahlreichen diagnostischen Überschneidungen und Abgrenzungsproblemen äußerst schwierig, eine exakte und sichere Diagnose zu stellen. Irrtümer bei der Diagnosestellung sind dementsprechend nicht die zwingende Folge eines vorwerfbaren Versehens des Arztes. Die Symptome einer Erkrankung sind nicht immer eindeutig, sondern können auf die verschiedensten Ursachen hinweisen. Das gilt auch unter Berücksichtigung der vielfachen technischen Hilfsmittel, die zur Gewichtung von zutreffenden Untersuchungsergebnissen einzusetzen sind. ${ }^{2}$ Erst recht sind vorläufige Diagnosen, wie sie etwa alsbald zum Zwecke der Entscheidung darüber gestellt werden müssen, ob der Patient eine Spezialbehandlung braucht, mit hohen Unsicherheitsfaktoren belastet. ${ }^{3}$ Diese Problematik hat auch die ständige Rechtspre-

2 BGH, Urt. v. 8.7.2003, Az.: VI ZR 304/02.

3 BGH, Urt. v. 14.7.1981, Az.: VI ZR 35/79.

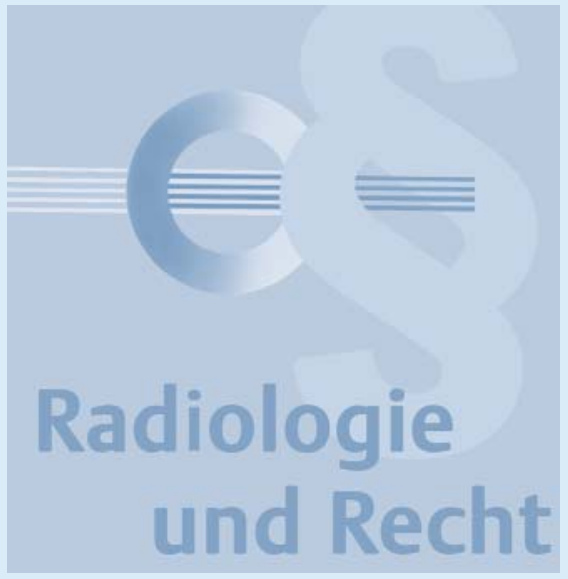

chung erkannt und ihr durch eine Haftungsprivilegierung Rechnung getragen. Die Gerichte ${ }^{4}$ sind daher sehr zurückhaltend bei der Annahme von Diagnosefehlern hinsichtlich der Fehlinterpretation von Befunden. Eine objektiv unrichtige Diagnose begründet folglich nicht notwendigerweise einen Behandlungsfehler.

Vor diesem Hintergrund ist zunächst zwischen dem bloßen Diagnoseirrtum und dem (vorwerfbaren) Diagnosefehler zu differenzieren. Ein Diagnoseirrtum liegt vor, wenn der Arzt erhobene Befunde oder sonst vorliegende Befunde falsch interpretiert und deshalb nicht die aus der berufsfachlichen Sicht seines Fachgebietes gebotenen therapeutischen oder diagnostischen Maßnahmen ergreift. Hinzutreten muss das Verschulden des Arztes, an welches gesteigerte Anforderungen in Form einer notwendigen Evidenz und eines gewissen Gewichtes des Diagnoseirrtums zu stellen sind. Nach der höchstrichterlichen Rechtsprechung ${ }^{5}$ muss die Schwelle eines „fundamentalen“ Diagnoseirrtums überschritten sein. Das Verschulden kann daher nur bei einer

- groben Verkennung der Diagnose oder

- unterbliebenen Überprüfung der Ver-

dachts- bzw. Arbeitsdiagnose angenommen werden. Im Ergebnis handelt es sich bei der Bewertung letztlich um eine Frage der medizinischen Vertretbarkeit der

4 Jüngst OLG Koblenz, Beschl. v. 20.2.2017, Az.: $5 \cup 1349 / 16$.

$5 \quad$ BGH, Urt. v. 9.1.2007, Az.: VI ZR 59/06; BGH, Urt. v. 12. 2.2008, Az.: VI ZR 221/06. 
gestellten Diagnose ${ }^{6}$. Dem Arzt obliegt es zudem, im Rahmen seiner Diagnosestellung auch der Eigendiagnose eines selbstbewusst auftretenden und vermeintlich selbst sachkundigen Patienten sowie etwaig von ihm geäußerten Therapiewünschen mit kritischer Distanz zu begegnen ${ }^{7}$.

Demgemäß erfordert der Diagnosefehler, dass der Arzt einen erhobenen oder sonst vorliegenden Befund vorwerfbar falsch interpretiert und deshalb (kausal) nicht die aus der berufsfachlichen Sicht seines Fachgebietes gebotenen therapeutischen oder diagnostischen Maßnahmen ergreift ${ }^{8}$.

\section{Fehlerhafte Fremddiagnosen}

Wird der Patient an einen Radiologen zur Leistungserbringung überwiesen (sog. horizontale Arbeitsteilung), so darf sich dieser zwar im Allgemeinen darauf verlassen, dass der überweisende Kollege des anderen Fachgebietes den Patienten in seinem Verantwortungsbereich mit der gebotenen Sorgfalt untersucht und behandelt hat und dass die Indikation zu der erbetenen Leistung zutreffend gestellt ist, solange keine offensichtlichen Qualifikationsmängel oder Fehlleistungen erkennbar werden ${ }^{9}$. Jedoch ist Zurückhaltung geboten: Die Pflicht zur Überprüfung und eigenständigen Meinungsbildung ist umso wichtiger, je schwerwiegender das Krankheitsbild ist. Hat der hinzugezogene Arzt indes aufgrund bestimmter Anhaltspunkte Zweifel an der Richtigkeit der ihm übermittelten Diagnose, muss er diesen Zweifeln nachgehen und darf sie nicht auf sich beruhen lassen. Das gilt insbesondere in den Fällen, in denen sich der überweisende Arzt an einen

6 Vgl. OLG Brandenburg, Urt. v. 21.7.2011, Az.: 12 U 9/11; OLG Jena, Urt. v. 6.3.2012, Az.: 4 U 26/11; OLG Naumburg, Urt. v. 31.5.2012, Az.: 1 U 97/11; OLG Koblenz, Urt. v. 21.11.2012, Az.: 5 U 381/12; OLG München, Urt. v. 8.8.2013, Az.: 1 U 4549/12; OLG Köln, Urt. v. 25.9.2013, Az.: 5 U 7/13; OLG Hamm, Urt.v. 21.1.2014, Az.: 26 U 101/12; OLG Koblenz, Beschl. v. 27.1.2014, Az.: 5 U 1383/13.

7 OLG Koblenz, Beschl. v. 30.1.2012, Az.: 5 U $857 / 11$.

8 BGH, Urt. v. 21.12.2010, Az.: VI ZR 284/09; BGH, Urt.v. 26.1.2016, Az.: VI ZR 146/14; OLG München, Urt. v. 8.8.2013, Az.: 1 U 4549/ 12.

$9 \quad$ BGH, Urt. v. 5.10.1993, Az.: VI ZR 237/92.
Facharzt wegen einer Leistung wendet, die er selbst nicht erbringen kann. ${ }^{10}$

In diesem Sinne urteilte auch das OLG $\mathrm{Hamm}^{11}$ in einer Entscheidung betreffend die ärztliche Aufgaben und Pflichten bei Überweisung einer Patientin vom Gynäkologen zum Radiologen wegen Brustkrebsverdachts. Erfolgt die Überweisung einer Patientin ausschließlich zur Durchführung einer bestimmten Untersuchung (hier Mammografie), ist der die Untersuchung durchführende Radiologe nicht zur umfassenden Beratung und Behandlung der Patientin verpflichtet. Eine Kontrolle des überweisenden Gynäkologen durch den Überweisungsempfänger - mithin den Radiologen - ist nach den Grundsätzen der „horizontalen Arbeitsteilung“ nur erforderlich, wenn sich Anhaltspunkte für ein fehlerhaftes Vorgehen ergeben.

\section{Abgrenzung zum Befund- erhebungsfehler}

Mit Blick auf die Haftungsprivilegierung im Rahmen des Diagnosefehlers sowie die Besonderheiten im Rahmen des Beweisrechtes (vgl. $§ 630$ h Abs. 5 S. 2 BGB) ist eine Abgrenzung zum Befunderhebungsfehler von besonderer Bedeutung. Ein Befunderhebungsfehler liegt vor, wenn medizinisch gebotene Befunde nicht erhoben wurden. Welche Befunde erhoben werden müssen, ist nach dem Stand der medizinischen Wissenschaft zu beurteilen. Dabei ist zu berücksichtigen, dass eine vollständige Abklärung sämtlicher theoretisch in Betracht kommender Erkrankungen regelmäßig nicht angezeigt ist. Insbesondere wenn die Befunderhebung den Patienten selbst einer Belastung oder Gefährdung aussetzt (z. B. Röntgenstrahlung, schmerzhafter Eingriff, Gefahr von Komplikationen etc.), ist der erwartete Nutzen in Verhältnis zu den Belastungen und Gefahren zu setzen. Der Nutzen bestimmt sich dabei unter Berücksichtigung des Ausmaßes der Ungewissheit, die durch die Befunderhebung abgeklärt werden soll, der Aussagekraft des zu erhebenden Befundes, der Wahrscheinlichkeit der abzuklärenden Erkrankung und dem Aus-

10 BGH, Urt. v. 5.10.1993, Az.: VI ZR 237/92; vgl. auch BGH, Urt. v. 14.7.1992, Az.: VI ZR 214/ 91.

11 OLG Hamm, Urt. v. 26.5.2005, Az.: 3 U 127/ 02 . maß der von ihr ausgehenden Gefahren für die Gesundheit des Patienten. ${ }^{12}$ Die Bejahung der Indikation zu einer risikobehafteten invasiven diagnostischen Maßnahme setzt eine besonders sorgfältige Güterabwägung zwischen der diagnostischen Aussagefähigkeit, den Klärungsbedürfnissen und den besonderen Risiken für den Patienten voraus ${ }^{13}$. Ebenso kann ein Übermaß an Diagnostik einen Behandlungsfehler darstellen ${ }^{14}$.

Es schließt sich jedoch die Frage an, welcher Behandlungsfehlertyp anzunehmen ist, wenn die fehlerhafte Diagnose zur Folge hat, dass keine weiteren Befunde erhoben werden. Insoweit kann sich die Unterscheidung vom Diagnosefehler zum Befunderhebungsfehler im Einzelfall als äußerst schwierig erweisen. Ein Diagnosefehler setzt voraus, dass der Behandler die medizinisch notwendigen Befunde überhaupt erhoben hat, um sich eine ausreichende Basis für die Einordnung der Krankheitssymptome zu verschaffen. Hat dagegen die unrichtige diagnostische Einstufung einer Erkrankung ihren Grund bereits darin, dass der Behandler die nach dem medizinischen Standard gebotenen Untersuchungen erst gar nicht veranlasst hat - er mithin aufgrund unzureichender Untersuchungen vorschnell zu einer Diagnose gelangt, ohne diese durch die medizinisch gebotenen Befunderhebungen abzuklären -, dann ist dem Arzt ein Befunderhebungsfehler vorzuwerfen. Denn bei einer solchen Sachlage geht es im Kern nicht um die Fehlinterpretation von Befunden, sondern um deren Nichterhebung. ${ }^{15}$ Dementsprechend liegt ein Befunderhebungsfehler vor, wenn der Arzt erkennt, dass das unklare Beschwerdebild des Patienten weitere differentialdiagnostische Maßnahmen (z. B. Hirndiagnostik) erfordert, er die wegen unzureichender Ausstattung der Klinik erforderliche Verlegung in ein ausreichend ausgestaltetes Krankenhaus aber auf den

12 Lafontaine, in: Herberger/Martinek/Rüßmann u. a., jurisPK-BGB, 8. Aufl. 2017, §630a Rn. 275 .

13 BGH, Urt. v. 4.4.1995, Az.: VI ZR 95/94.

14 Lafontaine, in: Herberger/Martinek/Rüßmann u. a., jurisPK-BGB, 8. Aufl. 2017, §630a Rn. 275.

15 BGH, Urt. v. 26.1.2016, Az.: VI ZR 146/14. 
nächsten Tag verschiebt ${ }^{16}$. Der mit der Diagnose befasste Arzt hat ferner die Pflicht, selbstkritisch die Grenzen seiner eigenen Erkenntnismöglichkeiten auf dem jeweiligen Fachgebiet zu erkennen und zu erwägen, dass angesichts der mannigfachen denkbaren Ursachen für eine Erkrankung oder Beschwerdesymptomatik der Arzt einer anderen Fachrichtung hinzugezogen werden muss. Ist dem Arzt aus differentialdiagnostischen Gründen eine eindeutige Zuordnung i.R. seines Fachgebietes gar nicht möglich und veranlasst er deswegen nicht die gebotene Abklärung durch andere Fachbereiche, liegt hierin kein Diagnosefehler, sondern ein Befunderhebungsfehler ${ }^{17}$.

Rechtlich problematisch an dieser Abgrenzung ist der Umstand, dass die - vorschnelle - Fehldiagnose (= Diagnosefehler) beweisrechtlich „belohnt“ wird, dokumentierte Unsicherheiten in der differentialdiagnostischen Zuordnung (= Befunderhebungsfehler) hingegen „bestraft“ werden. Die Lösung dieses Konfliktes ist in der Literatur $^{18}$ umstritten und gerichtlich noch nicht explizit entschieden. Die Judikatur ${ }^{19}$ stellt lediglich auf den Schwerpunkt der Vorwerfbarkeit ab: Fehlinterpretation eines Befundes oder Unterlassen der Befunderhebung. Stellt der Arzt eine objektiv unrichtige Diagnose, die aber ex ante noch vertretbar erscheinen durfte, stellt dies demgemäß keinen Befunderhebungsfehler dar, wenn Befunde nicht erhoben werden, die nur bei objektiv richtiger Diagnose zu erheben gewesen wären ${ }^{20}$, denn ein Diagnosefehler wird nicht dadurch zu einem Befunderhebungsfehler, dass bei objektiv zutreffender Diagnosestellung noch weitere Befunde zu erheben gewesen wären. Anderenfalls würde die Haftungsprivilegierung

16 BGH, Urt. v. 21.1.2014, Az.: VI ZR 78/13.

17 OLG München, Urt. v. 6.10.2011, Az.: 1 U 5220/10; OLG Koblenz, Beschl. v. 30.1.2012, Az.: $5 \cup 857 / 11$.

18 Vgl. Ramm, GesR 2011, 513; Hausch, MedR 2012, 231; Geiß/Greiner, Arzthaftpflichtrecht, 7. Aufl. 2014, Rn. 64.

19 Vgl. etwa OLG Hamm, Beschl. v. 2.3.2011, Az.: 3 U 92/10; OLG München, Urt. v. 6.10.2011, Az.: 1 U 5220/10; OLG Schleswig, Urt. v. 31.1.2013, Az.: 4 U 132/11; OLG Koblenz, Urt. v. 23.4.2014, Az.: 5 U 1427/13.

20 OLG Köln, Urt. v. 20.7.2005, Az.: 5 U 200/04; OLG Koblenz, Beschl. v. 26.9.2012, Az.: 5 U $783 / 12$. bei Diagnosefehlern stets unterlaufen, wenn Diagnoseirrtümer zu Befunderhebungsfehlern „umetikettiert“ werden ${ }^{21}$.

Zu beachten ist weiterhin, dass der Arzt auch vor Zufallsbefunden, die nicht hätten erhoben werden müssen, die Augen nicht verschließen darf. In diesem Kontext gelten die Fachgebietsgrenzen nur eingeschränkt: Übersieht ein Anästhesist eine ca. 2 Bildzentimeter durchmessende Verdichtungszone rechts supradiaphragmal (Rundherd) auf einem Röntgenbild, obwohl dies auch für ein ungeübtes Auge erkennbar gewesen wäre, und unterlässt deshalb eine weitere differentialdiagnostische Abklärung, liegt (primär) eine Fehlinterpretation des erhobenen Befundes, mithin ein Diagnosefehler vor ${ }^{22}$.

Nicht abschließend entschieden ist in der Rechtsprechung bis dato, ob es einen Diagnosefehler darstellt, wenn sich der Arzt in objektiv zweifelhaften Fällen vor endgültiger Diagnosestellung nicht der Richtigkeit seines Ergebnisses durch Einholung einer zweiten Meinung eines Kollegen versichert. Hier dürfte zu unterscheiden sein: Wenn der Behandler erkennen muss, dass sein eigenes Fachwissen und seine Erfahrung für eine hinreichend sichere Diagnose nicht ausreicht und ein Kollege über besseres Fachwissen oder breitere Erfahrung verfügt, ist die Zuziehung des Kollegen geboten. Verfügt der Behandler aber über ausreichende Kenntnisse und Erfahrungen, muss er von der bloßen Verdoppelung der Diagnosestellung keinen Mehrwert erwarten und kann diese unterlassen.

\section{Anforderungen an eine Mammo- grafie-Screening-Untersuchung}

Vor diesem Hintergrund stellt sich die Frage, welche Anforderungen an die Durchführung einer Mammografie-Screening-Untersuchung zu stellen sind. Die Anlage 9.2 zum BMV-Ä regelt die Versorgung im Rahmen des Programms zur Früherkennung von Brustkrebs durch Mammografie-Screening. Der Versorgungsauftrag umfasst gem. §3 Abs. 1 S. 2 Anlage 9.2 BMV-Ä die notwendige ärztliche Behandlung und

21 BGH, Urt. v. 21.12.2010, Az.: VI ZR 284/09; OLG Koblenz, Beschl. v. 6.7.2015, Az.: 5 U $347 / 15$.

22 BGH, Urt. v. 21.12.2010, Az.: VI ZR 284/09.
Betreuung der Patientinnen - insbesondere die Erstellung von Mammografie-Screeningaufnahmen sowie die Organisation und Durchführung der anschließenden Befundung nach $\S 10$ Anlage 9.2 BMV-Ä (§3 Abs. 3 lit. c und d Anlage 9.2 BMV-Ä) - einschließlich Aufklärung und Information sowie die übergreifende Versorgungsorganisation und -steuerung. Dieser Versorgungsauftrag wird durch den PVA übernommen, der hierfür der Genehmigung durch die Kassenärztliche Vereinigung bedarf (§4 Abs. 1 Anlage 9.2 BMV-Ä). Ihm obliegt es, die Doppelbefundung der durch eine radiologische Fachkraft unter verantwortlicher Leitung des PVA erstellten Screeningmammografieaufnahmen ( $§ 9$ Abs. 1 Anlage 9.2 BMV-Ä) gem. Abschnitt B Nr. 4 lit. g Abs. 1 der Krebsfrüherkennungsrichtlinien zu organisieren und in bestimmten Fällen eine zusätzliche Befundung vorzunehmen, §10 Abs. 1 Anlage 9.2 BMV-Ä. Hat mindestens ein Befunder den Befund als „auffällig und Abklärungsbedarf" eingestuft, wird der Fall in der Konsensuskonferenz eingebracht und kollegial beraten. Ziel der Konsensuskonferenz ( $§ 11$ Abs. 2 und 3 Anlage 9.2 BMV-Ä) ist es, eine abschließende, möglichst einheitliche Beurteilung der Screeningmammografieaufnahmen vorzunehmen. Bestehen trotz eingehender kollegialer Beratung unterschiedliche Auffassungen, legt der PVA die abschließende Beurteilung fest gem. $\S 11$ Abs. 4 Anlage 9.2 BMV-Ä.

Somit schließt sich die Frage an, ob die fehlende körperliche Untersuchung der Patientin durch den PVA bei unklarem Befund den Vorwurf eines Befunderhebungsfehlers rechtfertigt. In Übereinstimmung mit dem OLG Köln ${ }^{23}$ ist eine dahingehende Verpflichtung des PVA zu verneinen. Die systematische Beschränkung auf den radiologischen Aspekt erklärt sich aus dem Bedürfnis, eine möglichst große Zahl von Frauen zu erfassen, und zwar in einem Stadium, wo sich regelmäßig noch keine klinischen Auffälligkeiten ergeben haben. Eine derartige Verpflichtung kann im Übrigen auch nicht dem Wortlaut der Anlage 9.2 BMV-Ä sowie der Krebsfrüherkennungsrichtlinie entnommen werden. Dementsprechend ist eine Behandlung, deren

23 OLG Köln, Beschl. v. 12.10.2012, Az.: 5 U 102 | 12. 
Gegenstand die Durchführung einer Mammografie i.R.e. Krebs-Früherkennungsprogramms bildet, standardgerecht, wenn

- eine Mammografie erfolgt,

- diese durch Ärzte beurteilt und

- der Patientin das Ergebnis mitgeteilt wird.

Erst wenn sich aus dem abschließenden $\mathrm{Er}$ gebnis der Screening-Mammografie der Verdacht auf eine maligne Erkrankung ergibt, ist die Frau zur diagnostischen Abklärung einzuladen, zu der dann auch eine klinische Untersuchung, ggf. weitergehende mammografische Untersuchungen, Ultraschalldiagnostik und Stanzbiopsie gehören. Insoweit ist es unerheblich, dass es sich bei dem Mammografie-Screening nicht um einen regelhaften Eingriff, sondern um eine Vorsorgeuntersuchung handelt. Ergibt sich hingegen kein Verdacht auf eine maligne Erkrankung, so kommt es nicht zur Abklärungsdiagnostik und - im Wege des Gegenschlusses - auch nicht zu einer klinischen Untersuchung. Um Haftungsrisiken zu vermeiden, empfiehlt es sich jedoch, die Patientinnen darauf hinzuweisen, wo der Sinn und die Grenzen einer Krebsvorsorgeuntersuchung liegen, d. h. Beschränkung auf ärztliche Beurteilung bei (diagnostizierten) unauffälligem Befund.

Von diesem Ergebnis ist strikt die Frage zu trennen, ob möglicherweise ein Diagnosefehler vorausgegangenen ist. Ein solcher liegt vor, wenn bei regelgerechter Auswertung des Mammografie-Screenings der Befund eines malignen Mammakarzinoms festgestellt worden wäre, was zu weiteren Untersuchungen in Form von Ultraschalluntersuchungen bzw. eines MRT geführt hätte (die histologische Untersuchung ergab den Befund eines ca. 3,0 × 2,0 × 2,0 Zentimeter großen Karzinoms. ${ }^{24}$. Dieser Diagnosefehler begründete im streitgegenständlichen Fall einen Schmerzensgeldanspruch, da bei regelgerechter Diagnose und weiteren Untersuchungen sowie einer frühzeitigen Behandlungen eine Entfernung der linken Brust hätte vermieden werden können. Zu

24 LG Düsseldorf, Urt.v. 3.9.2015, Az.: 30 400/ 11; vgl. auch LG Dortmund, Urt. v. 17.3.2016, Az.: 4 O 210/11: fehlerhafte Einstufung der Mammografiebilder als „unauffällig“ (It. Sachverständigen-gutachten: BI-RADS 4); es hätte zudem überlegt werden können, ob Voraufnahmen beigezogen werden. beachten ist allerdings, dass außerhalb der Konsensuskonferenz kein Anspruch gegen den PVA besteht, da dieser nach $\S 10$ Abs. 4 S. 2 Anlage 9.2 BMV-Ä nur dann zur Befundung verpflichtet ist, wenn kein Konsens zwischen den beiden Vorbefundern besteht.

Mit Blick auf die Ausführungen zum Befunderhebungsfehler kann jedoch weder der Vorwurf eines Diagnoseirrtums noch der eines Diagnosefehlers, infolgedessen eine weitere Abklärungsdiagnostik unterbleiben ist, einen Befunderhebungsfehler wegen unterlassener körperlicher Untersuchung begründen (Stichwort: keine „Umetikettierung“).

\section{Haftung wegen Aufklärungsfehlern}

Besondere Anforderungen an den Umfang der Aufklärung 25 nach $§ 630$ e Abs. 1 BGB stellt die Rechtsprechung bei diagnostischen Eingriffen. Sie unterscheidet zwischen diagnostischen Eingriffen mit und ohne therapeutischen Eigenwert (z. B. Koronarangiografie, Endoskopie). Bei diagnostischen Eingriffen ohne therapeutischen Eigenwert - wie beispielsweise einer digitalen Subtraktionsangiografie des Kopfes ${ }^{26}$ - gelten allgemein strengere Maßstäbe für die Aufklärung des Patienten über die mit der medizinischen Maßnahme verbundenen Gefahren, sofern der invasive Schritt nicht gerade dringend oder sogar vital indiziert erscheint. Je weniger dringlich der diagnostische Eingriff ist, desto höher sind die an den Aufklärungsumfang zu stellenden Anforderungen. Bei ihnen bedarf es einer besonders sorgfältigen Abwägung zwischen der diagnostischen Aussagekraft, den Klärungsbedürfnissen und den besonderen Risiken für den Patienten ${ }^{27}$ sowie einer umfassenden Aufklärung des Patienten über Notwendigkeit, Zweckmäßigkeit und die Art der Risiken. Weiterhin hat der Arzt dem Patienten selbst entfernt liegende Kompli-

25 Eingehend zum Inhalt und Umfang der Aufklärungspflichten bei diagnostischen Röntgenuntersuchungen Wigge/Loose, Fortschr Röntgenstr 2016, S. 218 - 224 sowie S. 312 315.

26 BGH, Urt. v. 18.11.2008, Az.: VI ZR 198/07.

27 BGH, Urt. v. 18.11.2008, Az.: VI ZR 198/07. kationsmöglichkeiten in angemessener Weise darzutun ${ }^{28}$ und darüber zu unterrichten, wie sicher die gestellte Arbeitsdiagnose ist (diagnostische Aussagekraft). Unter Umständen besteht auch die Pflicht zur Einbeziehung und Berücksichtigung von Vorbefunden oder Vorerkrankungen.

Ist hingegen der diagnostische Eingriff beispielsweise wegen Malignitätsverdacht - vital oder bedingt vital indiziert, kann dies zwar den Aufklärungsumfang beschränken, ändert aber nichts an der grundsätzlichen Notwendigkeit, die Risiken des diagnostischen Eingriffs dem Patienten in angemessener Weise darzulegen ${ }^{29}$. Richtet sich der diagnostische Eingriff vorrangig auf Heilung oder Linderung und nur zugleich auch auf diagnostische Zwecke, so folgt der Grad der erforderlichen Aufklärung den Gegebenheiten des therapeutischen Eingriffs, beispielsweise bei der Hirnarteriografie, der Myelografie, der Koronarangiografie, der Nierenbiopsie und der Endoskopie ${ }^{30}$.

Beim Mammografie-Screening gelten dieselben rechtlichen Maßstäbe, wie bei „sonstigen" diagnostischen Eingriffen, da das Gesetz nicht zwischen beiden differenziert: Beide stellen eine Behandlung i. S. d. §630a Abs. 1 BGB dar. Demzufolge gilt uneingeschränkt der Pflichtenkanon des §630e Abs. 1 BGB. Insoweit ist es wiederum unerheblich, dass es sich bei dem Mammografie-Screening nicht um einen regelhaften Eingriff, sondern um eine Vorsorgeuntersuchung handelt. Daher sollte die Patientin vor der Durchführung des Screenings über dessen Erfolgsaussichten aufgeklärt und der prognostische Hinweis gegeben werden, wie wahrscheinlich "falsch negative“ und „falsch positive“ Befunde sind. Bei dem Aufklärungsgespräch handelt es sich um ein freiwilliges Angebot. Um Haftungsrisiken zu vermeiden, sollte die Patientin daher im Falle eines Verzichtes eine entsprechende Erklärung unterschreiben

28 Vgl. Laufs, in: Laufs/Kern (Hrsg), Handbuch des Arztrechts, 4. Aufl. 2010, §60 Rnrn. 8 ff.; BGH, Urt. v. 15.5.1979, Az.: VI ZR 70/77; OLG Karlsruhe, Urt. v. 2.3.1988, Az.: 7 U 2/84; OLG Koblenz, Urt. v. 29.11.2001, Az.: 5 U 1382/00. BGH, Urt. v. 15.5.1979, Az.: VI ZR 70/77.

30 Vgl. Laufs, in: Laufs/Kern (Hrsg), Handbuch des Arztrechts, 4. Aufl. 2010, §60 Rn. 10. 
und zum Termin mitbringen. Seitdem 1.10.2016 besteht nunmehr auch die Möglichkeit der gesonderten Abrechnung des Aufklärungsgesprächs i.R.d. MammografieScreenings (GOP 01751 , Aufklärungsgespräch im Rahmen des Programms zur Früherkennung von Brustkrebs durch Mammografie-Screening). Voraussetzungen der Abrechnungsziffer sind:

- Gespräch muss vor der Röntgenuntersuchung beider Mammae (GOP 01750 ) erfolgen und soll über Hintergründe, Ziele, Inhalte und Vorgehensweise des Früherkennungsprogramms informieren

- Persönlicher Arzt-Patienten-Kontakt erforderlich

- Je vollendete 5 Minuten berechnungsfähig

- Dreimal im Krankheitsfall berechnungsfähig

- Bei Abrechnung der GOP 01751 und 01750 am selben Behandlungstag sind die Uhrzeiten in der Abrechnung anzugeben

- Nur vom PVA oder einem durch ihn beauftragten Arzt berechnungsfähig.

\section{Fazit}

Da die Judikatur dem Arzt bei Diagnosefehlern eine Haftungsprivilegierung zubilligt, ist die Abgrenzung zum Befunderhebungsfehler von entscheidender Bedeutung. Diese kann, wie der Beitrag gezeigt hat, im Einzelfall jedoch schwierig sein. Hinsichtlich der Aufklärung von Patienten bei diagnostischen Eingriffen bestehen gesteigerte Anforderungen. Diese Wertungen lassen sich uneingeschränkt auf die Bestimmung des Pflichtenkanons beim MammografieScreening übertragen. Dabei ist zu beachten, dass weder PVA noch die übrigen mit dem Screening befassten Ärzte bei einem Befund, den sie als „unauffällig“ bewertet haben, zur körperlichen Untersuchung verpflichtet sind. Um Haftungsrisiken zu vermeiden, ist gleichwohl anzuraten, die Patientinnen darauf hinzuweisen, wo der Sinn und die Grenzen einer solchen Krebsvorsorgeuntersuchung liegen.
Prof. Dr. Peter Wigge

Rechtsanwalt

Fachanwalt für Medizinrecht

Dipl. jur. Jan Harald Schütz, LL.M., Wiss. Mitarbeiter

Rechtsanwälte Wigge

Scharnhorststraße 40

48151 Münster

$++49 / 251 / 535950$

$++49 / 251 / 5359599$

kanzlei@ra-wigge.de

www.ra-wigge.de 